\title{
Dynamic evolution of pairs of trans-Neptunian objects: the case of binary and single objects in pair
}

\author{
E. Kuznetsov, O. Al-Shiblawi and V. Gusev \\ Ural Federal University \\ Lenina Avenue, 51, Yekaterinburg, 620000 Russian Federation (E-mail: \\ eduard.kuznetsov@urfu.ru)
}

Received: August 1, 2021; Accepted: October 20, 2021

\begin{abstract}
We performed a search for pairs of trans-Neptunian objects in close orbits with semi-major axes of more than $30 \mathrm{AU}$. Distances in the space of Keplerian orbits were estimated using the Kholshevnikov metrics. Found 21 pairs of trans-Neptunian objects in which one of the components is binary, for Kholshevnikov metrics in five-dimensional space of Keplerian orbits less than $0.121 \mathrm{AU}^{1 / 2}$. All pairs belong to cold Classical Kuiper belt objects. Based on nominal orbits, the dynamic evolution of pairs of trans-Neptunian objects in the past 10 Myr has been studied numerically. We searched for low relativevelocity close encounters between trans-Neptunian objects in pairs as well as the minima of the Kholshevnikov metrics and the convergence of the lines of nodes and apses, to estimate the age of the pairs.
\end{abstract}

Key words: Trans-Neptunian objects - Classical Kuiper belt objects - celestial mechanics

\section{Introduction}

Apart from Pluto and Charon, the first trans-Neptunian object (TNOs) was found in 1992. Although many TNOs were found on quite elliptic orbits, some of them had roughly circular orbits on a plane near the ecliptic (or the invariant solar system plane), today about 3500 objects have been recognized and indexed. The distribution of the orbits of asteroids in the Solar System is the result of various processes that affect for a long time (see e.g. Deienno et al., 2016; Granvik et al., 2017).

As was shown in (Vokrouhlický \& Nesvorný, 2008), in the main belt of asteroids, there are a large number of pairs of asteroids with close orbits that have a common origin. A study of these pairs (Pravec \& Vokrouhlický, 2009) proved their statistical significance. Unbound pairs of asteroids have also been identified in (Pravec et al., 2010; Jacobson, 2016; Kuznetsov \& Safronova, 2018). One candidate pair resides in the scattered disc (Rabinowitz et al., 2011). Groups of pairs define young asteroid clusters (Pravec et al., 2018; Kuznetsov \& Vasileva, 2019). 
Different processes can lead to the formation of pairs or groups of minor bodies with close orbits. It is collisional break-ups, rotational or thermal-stressinduced splittings, tidal disruptions, and binary dissociations (see e.g., (Benz \& Asphaug, 1999; Boehnhardt, 2004; Sekanina \& Chodas, 2005, 2007; Bottke et al., 2006; Jacobson \& Scheeres, 2011; Schunová et al., 2014; Jacobson, 2016; Vokrouhlický et al., 2017). Mean motion and secular resonances can also induce orbital coherence (see e.g. de la Fuente Marcos \& de la Fuente Marcos, 2016).

A candidate collisional family in the outer Solar system was proposed by Chiang (2002). The first asteroid family identified in the outer Solar system was the one associated with dwarf planet Haumea (Brown et al., 2007). The subject of finding collisional families of trans-Neptunian objects has been studied by Chiang et al. (2003) and Marcus et al. (2011). de la Fuente Marcos \& de la Fuente Marcos (2018) perform a systematic search for statistically significant pairs and groups of dynamically correlated objects through those with a semimajor axis greater than $25 \mathrm{AU}$, applying a technique that uses the angular separations of orbital poles and perihelia together with the differences in time of perihelion passage to single out pairs of relevant objects from which groupings can eventually be uncovered. They confirm the reality of the candidate collisional family of TNOs associated with the pair $2000 \mathrm{FC}_{8}-2000 \mathrm{GX}_{146}$ and initially proposed by Chiang (2002). They find four new possible collisional families of TNOs associated with the pairs (134860) $2000 \mathrm{OJ}_{67}-2001 \mathrm{UP}_{18}, 2003 \mathrm{UT}_{291}-$ $2004 \mathrm{VB}_{131}, 2002 \mathrm{CU}_{154}-2005 \mathrm{CE}_{81}$ and $2003 \mathrm{HF}_{57}-2013 \mathrm{GG}_{137}$. They find several unbound TNOs that may have a common origin, the most significant ones are (135571) $2002 \mathrm{GG}_{32}-(160148) 2001 \mathrm{KV}_{76}$ and $2005 \mathrm{GX}_{206}-2015$ $\mathrm{BD}_{519}$.

In (Kuznetsov et al., 2021) performed a search for statistically significant pairs and groups of dynamically correlated objects through those with a semimajor axis greater than $30 \mathrm{AU}$, applying a novel technique that uses Kholshevnikov metrics (Kholshevnikov et al., 2016, 2020) in the space of Keplerian orbits. Found 27 pairs of TNOs in close orbits, 22 pairs in which one of the TNO is binary, and 11 pairs of binary trans-Neptunian objects. All pairs belong to cold classical Kuiper belt objects. Among the dynamically cold population of the classical Kuiper belt, during the evolution of the protoplanetary disk and the migration of planets, conditions are implemented for the preservation of close binary or contact TNOs with components of approximately equal masses (Nesvorný \& Vokrouhlický, 2019). On the other hand, the evolution of wide binary trans-Neptunian objects turns out to be unstable due to frequent encounters with other TNOs, which lead to the decay of binary systems (Campbell, 2021) and the formation of TNO pairs in close orbits.

We perform a study of the dynamical evolution of pairs of TNOs in which one of the component is binary. This paper is organized as follows. Section 2 reviews the methods which we used to search for TNOs with close orbits, research the dynamical evolution of TNOs, and estimate the age of the TNO pairs. Some 
pairs found by our approach are presented in Section 3. In Section 4, we discuss the results and summarize our conclusions.

\section{Methods}

We have used natural metrics $\varrho\left(\mathcal{E}_{1}, \mathcal{E}_{2}\right)$ in the space of Keplerian orbits (Kholshevnikov et al., 2016, 2020) to search for TNOs with close orbits. Here Keplerian orbits $\mathcal{E}_{s}$ are points in a five-dimensional space of orbits (the position on the orbit is omitted). Let us denote by $a, p, e, i, \omega, \Omega$ the semi-major axis, semilatus rectum, eccentricity, inclination, argument of the pericentre and longitude of the ascending node of the orbit, respectively.

The metric $\varrho_{2}$ defines the distance between two orbits in the five-dimensional space of Keplerian orbits (Kholshevnikov et al., 2016)

$$
\varrho_{2}^{2}=\left(1+e_{1}^{2}\right) p_{1}+\left(1+e_{2}^{2}\right) p_{2}-2 \sqrt{p_{1} p_{2}}\left(\cos I+e_{1} e_{2} \cos P\right),
$$

here indices 1 and 2 correspond to orbital elements of the first and second bodies, respectively,

$$
\begin{gathered}
\cos I=c_{1} c_{2}+s_{1} s_{2} \cos \Delta, \\
\cos P=s_{1} s_{2} \sin \omega_{1} \sin \omega_{2}+ \\
\left(\cos \omega_{1} \cos \omega_{2}+c_{1} c_{2} \sin \omega_{1} \sin \omega_{2}\right) \cos \Delta+ \\
\left(c_{2} \cos \omega_{1} \sin \omega_{2}-c_{1} \sin \omega_{1} \cos \omega_{2}\right) \sin \Delta, \\
c=\cos i, \quad s=\sin i, \quad \Delta=\Omega_{1}-\Omega_{2} .
\end{gathered}
$$

The metric $\varrho_{5}$ defines the distance in the three-dimensional factor-space of the positional elements. Its elements are classes of orbits with fixed $p, e, i$ and all possible values of $\omega$ and $\Omega$ (Kholshevnikov et al., 2016)

$$
\varrho_{5}^{2}=\left(1+e_{1}^{2}\right) p_{1}+\left(1+e_{2}^{2}\right) p_{2}-2 \sqrt{p_{1} p_{2}}\left\{e_{1} e_{2}+\cos \left(i_{1}-i_{2}\right)\right\} .
$$

The metric $\varrho_{2}$ shows the current distance between the Keplerian orbits. The metric $\varrho_{5}$ gives the minimum distance between the orbits among all possible positions of the nodes and pericenter of the orbits. Analyzing the metrics will help identify candidates for young pairs. The positions of the lines of nodes and apses of the TNO orbits in young pairs should be close because the orientation of the orbits has changed slightly since the formation of the pair due to the secular drift of nodes and pericenter. If the metrics $\varrho_{2}$ and $\varrho_{5}$ are small (for TNO pair with binary, one can limit ourselves to $0.12 \mathrm{AU}^{1 / 2}$ ) and have close values (e.g. $\varrho_{2}-\varrho_{5}<0.025 \mathrm{AU}^{1 / 2}$ ), then such a pair of TNOs can be considered a candidate for young pair (e.g Kuznetsov et al., 2020).

We have used both numbered and multiopposition objects from the Asteroids Dynamic Site (AstDyS, https://newton.spacedys.com/astdys/). Epoch of the orbital elements is MJD $58800\left(00^{h} 00^{m} 00.000^{s}\right.$ BDT 13.11.2019) depending 
on the used sets of orbital elements from the AstDyS. The metrics $\varrho_{2}(1)$ and $\varrho_{5}(2)$ have been calculated to search for TNOs pairs with close orbits.

The dynamic evolution of TNO pairs was studied in two stages. In the first step, to find close approaches of TNOs in pairs in the past and, therefore, estimate the age of the pairs, we have performed numerical integrations of the orbits of TNOs in pairs backward in time (a time span of $10 \mathrm{Myr}$ ) with the code known as Orbit9 (the OrbFit Software Package, http://adams.dm.unipi.it/orbfit/ (Orbfit Consortium, 2011)). Orbit9 software allows us to estimate the maximum Lyapounov Characteristic Exponent (LCE). We estimated the LCE for the cold classical Kuiper belt to be $0.1 \mathrm{Myr}$ that makes it possible to study the evolution of nominal orbits over several million years. The numerical integrations were made taking the nominal orbits given by AstDyS database as initial conditions. The eight major planets and the dwarf planet Pluto were integrated consistently. The mean ecliptic of J2000.0 was taken as reference plane for the output. We used heliocentric coordinates.

The standard methods for determining the age of pairs of small bodies in close orbits include analysis of 1) low relative-velocity close encounters of objects (see, e.g. Pravec et al., 2019), 2) the minimum distances between the orbits of objects (see, e.g. Kuznetsov et al., 2020), 3) simultaneous approaches of node lines and apse lines of objects orbits (see, e.g. Rosaev \& Plávalová, 2017).

The analysis of simultaneous approaches of the lines of nodes and apse of the TNO orbits can be considered as a special case of a more general method using the analysis of the minimum distances between the TNO orbits, for example, using the Kholshevnikov metrics. When analyzing the simultaneous approaches of the lines of nodes and lines of the apses, only two elements are used the longitude of the ascending node and the argument of the pericenter. At the same time, the estimate of the minimum distance between the orbits is calculated based on five Keplerian elements - the semi-major axis, eccentricity, inclination, longitude of the ascending node, and the argument of the pericenter. Since at the moment of pair formation, the orbits of the objects are close, the difference in the longitudes of the ascending nodes $\Delta \Omega$ and the difference in the arguments of the pericenter $\Delta g$ should vanish simultaneously with the attainment of a minimum by the metric $\varrho_{2}$.

The condition of convergence of orbits does not yet guarantee the convergence of objects moving in these orbits. Therefore, to estimate the age of pairs, it is also necessary to analyze the possibility of the onset of low relative-velocity close encounters, at which the distance between objects $r_{r e l}$ is comparable to the radius of the Hill sphere $R_{H}$ of a more massive body, and the relative velocity $v_{r e l}$ is of the order of the escape velocity $V_{e s c}$ relative to a more massive body. Pravec et al. (2019) used the criteria for low-speed encounters for asteroids in the main asteroid belt: $r_{r e l}<(5$ or 10$) R_{H}, v_{r e l}<(2$ or 4$) V_{e s c}$, where $V_{e s c}$ is the escape velocity on the surface of a more massive body. The narrower limits were used for better converging orbits (e.g., younger ones, or those in non-chaotic 
zones of the main asteroid belt), while the loosened limits were typically used for pairs with the orbits affected by some orbital chaoticity.

For each close approach of TNOs in pair we determined the relative distance $r_{r e l}$ between TNOs and relative velocity $v_{r e l}$, as well as the Hill sphere radius $R_{H}$ and escape velocity $V_{e s c}$ of the primary body. The radius of the Hill sphere was estimated as:

$$
R_{H}=\frac{1}{2} r_{1} D_{1}\left(\frac{4 \pi}{9} \frac{G \rho_{1}}{\mu}\right)^{1 / 3}
$$

where $r_{1}$ is the heliocentric distance of the primary's TNO, $D_{1}$ is its diameter, $\rho_{1}$ is its bulk density, $G$ is the gravitational constant and $\mu$ is the gravitational parameter of the Sun. The escape velocity of primary body for relative distance $r_{r e l}$ was estimated as:

$$
V_{e s c}=\sqrt{\frac{\pi}{3} \frac{G D_{1}^{3} \rho_{1}}{r_{r e l}}} .
$$

The diameter $D$ of the TNO can be estimated from the absolute magnitude $H$ and the geometric albedo $p_{v}$ (Bowell et al., 1989):

$$
D=1329 \mathrm{~km} 10^{-H / 5} \frac{1}{\sqrt{p_{v}}} .
$$

We need to know the physical parameters of the TNO to estimate the radius of the Hill sphere $R_{H}$ (3) and the escape velocity $V_{\text {esc }}$ (4). Since the objects included in the studied TNO pairs belong to the dynamically cold population of the classical Kuiper belt and have dimensions not exceeding several hundred km, we used the same density values $\rho=0.5 \mathrm{~g} \mathrm{~cm}^{-3}$ and geometric albedo $p_{v}=0.13$ for all TNOs (Muller et al. ., 2020).

Estimates of the single TNO density range from 0.5 to $2 \mathrm{~g} \mathrm{~cm}^{-3}$ (Lacerda \& Jewitt, 2007; Grundy et al., 2008; Fernández, 2020) and grow with an increase in the TNO diameter. For TNOs several hundred $\mathrm{km}$ in size, the density estimates are $0.5-0.6 \mathrm{~g} \mathrm{~cm}^{-3}$ (Lacerda \& Jewitt, 2007; Grundy et al., 2008; Fernández, 2020). We used the minimum density value $\rho=0.5 \mathrm{~g} \mathrm{~cm}^{-3}$, which will give the minimum estimates for the radius of the Hill sphere $R_{H}$ (3) and the escape velocity $V_{\text {esc }}$ (4). If the density value is $2 \mathrm{~g} \mathrm{~cm}^{-3}$, the value of the radius of the Hill sphere $R_{H}$ will be underestimated by $4^{1 / 3} \approx 1.6$ times, and the value of the escape velocity $V_{\text {esc }}$ by $4^{1 / 2}=2$ times. This can be taken into account when establishing the criteria for close encounters of the TNO pair. We used the physical parameters of binary TNOs published in (Grundy et al., 2009, 2011, 2019) 


\section{Results}

\subsection{Search for pairs with binary TNO in close orbits}

We used the osculating orbital elements from the AstDyS database for the epoch MJD 58800, to calculate the Kholshevnikov metrics $\varrho_{2}(1)$ and $\varrho_{5}(2)$. The following criteria were chosen for the selection of young pairs of TNOs: $\varrho_{2} \leq 0.121 \mathrm{AU}^{1 / 2}\left(\varrho_{2}^{2} \leq 0.0146 \mathrm{AU}=2.2 \cdot 10^{6} \mathrm{~km}\right), \varrho_{5}<0.12 \mathrm{AU}^{1 / 2}\left(\varrho_{5}^{2}<\right.$ $\left.0.0144 \mathrm{AU}=2.2 \cdot 10^{6} \mathrm{~km}\right)$ and $\varrho_{2}-\varrho_{5}<0.025 \mathrm{AU}^{1 / 2}\left(\left(\varrho_{2}-\varrho_{5}\right)^{2}<0.000625 \mathrm{AU}\right.$ $\left.=9.4 \cdot 10^{4} \mathrm{~km}\right)$. The criteria for the metrics $\varrho_{2}$ and $\varrho_{5}$ correspond to two or three values of the Hill sphere radius for TNO. The closeness of the metrics $\varrho_{2}$ and $\varrho_{5}$ may indicate the youth of a pair of TNOs (after forming a pair, the orbits have not yet had time to disperse due to the precession of nodes and pericenter). However, this is only a necessary condition for the youth of the pairs because the precession of the nodes and pericenter of the orbits has a conditionally periodic type.

We selected 21 pairs of TNOs with binaries satisfying the first two conditions: $\varrho_{2} \leq 0.121 \mathrm{AU}^{1 / 2}$ and $\varrho_{5}<0.12 \mathrm{AU}^{1 / 2}$ (see Tab. 1 ). Ten pairs of TNOs (in bold in Tab. 1) satisfy all three conditions. Tab. 2 gives the orbital elements for the epoch MJD 58800, here $M_{0}$ is the mean anomaly, and $H$ is the absolute magnitude of TNO. Analysis of the Tab. 2 shows that all detected TNOs included in the pairs belong to the dynamically cold population of the classical Kuiper belt, which is characterized by orbits with semi-major axes from 42 to $45 \mathrm{AU}$, eccentricities not exceeding 0.1 , and inclinations not exceeding $5^{\circ}$ (Gladman et al., 2008; Kavelaars et al., 2008). The minimum value of the semi-major axis for TNO $2003 \mathrm{UN}_{284}$ is $42.4 \mathrm{AU}$, the maximum value for 2015 $\mathrm{RP}_{280}$ is $45.5 \mathrm{AU}$. The maximum eccentricity of 0.105 has TNO $2012 \mathrm{HE}_{85}$. The maximum inclination of $3.8^{\circ}$ is achieved for TNO $2003 \mathrm{QY}_{90}$.

\subsection{Dynamic evolution of pairs with binary TNO in close orbits}

We carried out a numerical simulation of the orbital evolution in the time interval of 10 Myr into the past for all selected pairs with binary TNO (see Tab. 1). The nominal values of the osculating elements of the TNO orbits from the AstDyS base for the epoch MJD 58800 (see Tab. 2) were chosen as the initial ones.

Tab. 3 shows the minimum values of the metric $\varrho_{2 m i n}$ and the corresponding moments $t_{\varrho}$, measured in years from the epoch MJD 58800. Tab. 3 gives the pairs in the same order as in Tab. 1. For 13 pairs, the minimum value of the metric is $\varrho_{2 \min }<0.07 \mathrm{AU}^{1 / 2}\left(\varrho_{2 \min }^{2}<0.0049 \mathrm{AU}=7.33 \cdot 10^{5} \mathrm{~km}\right)$. The minimum value $\varrho_{2 \min }=0.0057 \mathrm{AU}^{1 / 2}\left(\varrho_{2 \min }^{2}=3.25 \cdot 10^{-5} \mathrm{AU}=4.86 \cdot 10^{3} \mathrm{~km}\right)$ was recorded for the pair TNO (469610) $2004 \mathrm{HF}_{79}-2013 \mathrm{UL}_{17}$.

The moments $t_{\varrho}$ corresponding to the minima of the metrics are not the moments of TNO pairs formation since, in addition to the approach of the orbits, the objects must also encounter. A search was carried out for low relativevelocity close encounters of TNOs included in pairs in close orbits (see Tab. 1), 
Table 1. TNOs pairs with binaries.

\begin{tabular}{|c|c|c|c|}
\hline 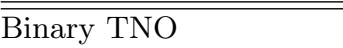 & "TNO & 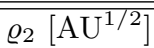 & 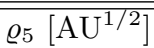 \\
\hline 2005 GD $_{187}$ & $2001 \mathbf{O G}_{109}$ & 0.0731 & 0.0664 \\
\hline$(275809) 2001 \mathrm{QY}_{297}$ & $2015 \mathbf{V B}_{169}$ & 0.0732 & 0.0654 \\
\hline (469610) $2004 \mathbf{H F}_{79}$ & $1997 \mathbf{C T}_{29}$ & 0.0791 & 0.0546 \\
\hline $2005 \mathrm{GD}_{187}$ & $2015 \mathrm{VV}_{170}$ & 0.0918 & 0.0664 \\
\hline $2002 \mathrm{VD}_{131}$ & $(505446) 2013 \mathrm{SP}_{99}$ & 0.0976 & 0.0945 \\
\hline $2004 \mathrm{HK}_{79}$ & $2015 \mathrm{VB}_{171}$ & 0.0996 & 0.0657 \\
\hline $2006 \mathrm{JV}_{58}$ & $2004 \mathrm{KF}_{19}$ & 0.0998 & 0.0707 \\
\hline (275809) $2001 \mathrm{QY}_{297}$ & $2006 \mathbf{H B}_{123}$ & 0.1039 & 0.0856 \\
\hline $2003 \mathrm{QY}_{90}$ & $2002 \mathrm{CU}_{154}$ & 0.1049 & 0.0957 \\
\hline $2015 \mathbf{R P}_{280}$ & $2012 \mathrm{HE}_{85}$ & 0.1083 & 0.0933 \\
\hline $2000 \mathbf{C F}_{105}$ & $2015 \mathbf{G J}_{57}$ & 0.1110 & 0.0977 \\
\hline $2003 \mathrm{UN}_{284}$ & $1996 \mathrm{TK}_{66}$ & 0.1124 & 0.0834 \\
\hline $2015 \mathrm{VW}_{168}$ & $2013 \mathrm{UL}_{17}$ & 0.1133 & 0.0989 \\
\hline $2004 \mathrm{HK}_{79}$ & $2004 \mathrm{VB}_{131}$ & 0.1135 & 0.0674 \\
\hline (505447) 2013 SQ99 & $2004 \mathrm{HE}_{79}$ & 0.1141 & 0.0872 \\
\hline $2004 \mathrm{HK}_{79}$ & $2015 \mathrm{GA}_{57}$ & 0.1144 & 0.0199 \\
\hline $2005 \mathrm{GD}_{187}$ & (420356) Praamzius & 0.1148 & 0.0889 \\
\hline $2004 \mathrm{PW}_{117}$ & $2015 \mathrm{GT}_{57}$ & 0.1174 & 0.0978 \\
\hline $2002 \mathrm{VD}_{131}$ & $2002 \mathrm{CZ}_{224}$ & 0.1184 & 0.0983 \\
\hline $2004 \mathrm{PW}_{117}$ & $2013 \mathrm{TL}_{172}$ & 0.1188 & 0.0265 \\
\hline (469610) $2004 \mathrm{HF}_{79}$ & $2013 \mathrm{UL}_{17}$ & 0.1209 & 0.0837 \\
\hline
\end{tabular}

based on the results of numerical modeling. The integration interval was $10 \mathrm{Myr}$ in the past. The nominal TNO orbits from the AstDyS database were used. The criteria for the search for low relative-velocity close encounters were chosen taking into account the uncertainty of the density $\rho$ and albedo $p_{v}$ of the TNO: $r_{r e l}<3 R_{H}, v_{r e l}<4 V_{e s c}$. On the considered time interval, for none of the pairs, the conditions of low relative-velocity close encounters were fulfilled.

Tab. 4 shows information about the approaches of TNO to distances less than $10 R_{H}$ (here $t_{r}$ is the moment at which the minimum distance is reached). There are only six such pairs. The closest approach of the TNOs to $r_{r e l}=2.8 R_{H}$ was recorded for the pair TNO $2005 \mathrm{GD}_{187}-(420356)$ Praamzius as the minimum relative velocity $v_{r e l}=167 V_{e s c}$. However, this velocity is 42 times higher than the value corresponding to the low relative-velocity close encounters. We can conclude that in the considered interval of $10 \mathrm{Myr}$, the encounters of TNOs to distances of less than $10 R_{H}$ is possible. Still, high speeds of relative motion show that the moments of close approaches of TNOs are most likely not the moments of formation of pairs of TNOs in close orbits. 
Table 2. Orbital elements of TNOs pairs with binaries at epoch MJD58800.0.

\begin{tabular}{|c|c|c|c|c|c|c|c|}
\hline$\overline{\overline{\mathrm{TNO}}}$ & $\begin{array}{l}a \\
{[\mathrm{AU}]}\end{array}$ & $e$ & $\begin{array}{l}i \\
{\left[{ }^{\circ}\right]}\end{array}$ & $\begin{array}{c}\Omega \\
{\left[{ }^{\circ}\right]}\end{array}$ & $\begin{array}{r}g \\
{\left[{ }^{\circ}\right]}\end{array}$ & $\begin{array}{c}M_{0} \\
{\left[^{\circ}\right]}\end{array}$ & $\begin{array}{l}H \\
{[\mathrm{mag}]}\end{array}$ \\
\hline$\overline{(275809) 2001 \mathrm{QY}_{297}}$ & 44.117 & 0.085 & 1.547 & 108.75 & 129.91 & 82.73 & 5.58 \\
\hline (420356) Praamzius & 42.531 & 0.011 & 1.101 & 314.26 & 1.46 & 182.67 & 5.60 \\
\hline (469610) $2004 \mathrm{HF}_{79}$ & 43.598 & 0.034 & 1.485 & 76.64 & 193.70 & 338.79 & 6.42 \\
\hline (505446) $2013 \mathrm{SP}_{99}$ & 43.805 & 0.062 & 0.791 & 71.92 & 250.51 & 52.07 & 7.36 \\
\hline (505447) $2013 \mathrm{SQ}_{99}$ & 44.181 & 0.094 & 3.469 & 51.96 & 177.15 & 142.02 & 6.57 \\
\hline $1996 \mathrm{TK}_{66}$ & 42.565 & 0.016 & 3.315 & 44.59 & 211.26 & 136.86 & 6.37 \\
\hline $1997 \mathrm{CT}_{29}$ & 43.643 & 0.034 & 1.014 & 74.52 & 210.21 & 239.25 & 6.56 \\
\hline $2000 \mathrm{CF}_{105}$ & 43.684 & 0.032 & 0.528 & 56.47 & 56.74 & 31.87 & 6.94 \\
\hline $2001 \mathrm{OG}_{109}$ & 43.759 & 0.018 & 0.540 & 332.70 & 1.58 & 355.71 & 8.15 \\
\hline $2002 \mathrm{CU}_{154}$ & 43.652 & 0.056 & 3.355 & 108.98 & 41.12 & 9.66 & 6.91 \\
\hline $2002 \mathrm{CZ}_{224}$ & 44.853 & 0.065 & 1.687 & 66.48 & 263.82 & 183.34 & 6.95 \\
\hline $2002 \mathrm{VD}_{131}$ & 45.006 & 0.066 & 0.851 & 84.62 & 239.38 & 70.85 & 6.57 \\
\hline $2003 \mathrm{QY}_{90}$ & 42.922 & 0.047 & 3.762 & 104.05 & 41.84 & 198.99 & 6.48 \\
\hline $2003 \mathrm{UN}_{284}$ & 42.411 & 0.004 & 3.069 & 35.99 & 277.08 & 121.47 & 7.42 \\
\hline $2004 \mathrm{HE}_{79}$ & 44.664 & 0.104 & 3.085 & 62.82 & 164.96 & 17.20 & 7.42 \\
\hline $2004 \mathrm{HK}_{79}$ & 44.179 & 0.085 & 1.943 & 69.02 & 182.48 & 340.69 & 6.92 \\
\hline $2004 \mathrm{KF}_{19}$ & 44.470 & 0.070 & 0.107 & 329.90 & 277.11 & 2.75 & 6.92 \\
\hline $2004 \mathrm{PW}_{117}$ & 43.868 & 0.055 & 1.862 & 81.35 & 76.57 & 167.84 & 6.34 \\
\hline $2004 \mathrm{VB}_{131}$ & 44.027 & 0.076 & 1.745 & 50.37 & 207.53 & 153.34 & 6.55 \\
\hline $2005 \mathrm{GD}_{187}$ & 42.971 & 0.022 & 0.707 & 354.40 & 333.59 & 200.83 & 7.21 \\
\hline $2006 \mathrm{HB}_{123}$ & 44.448 & 0.097 & 1.801 & 115.76 & 128.02 & 5.93 & 7.04 \\
\hline $2006 \mathrm{JV}_{58}$ & 45.323 & 0.067 & 0.318 & 144.15 & 95.97 & 11.25 & 6.63 \\
\hline $2012 \mathrm{HE}_{85}$ & 44.926 & 0.105 & 3.016 & 235.00 & 38.36 & 12.37 & 9.04 \\
\hline $2013 \mathrm{TL}_{172}$ & 43.833 & 0.058 & 1.791 & 95.04 & 78.81 & 197.66 & 6.85 \\
\hline $2013 \mathrm{UL}_{17}$ & 42.712 & 0.030 & 1.831 & 82.27 & 211.10 & 86.11 & 8.17 \\
\hline $2015 \mathrm{GA}_{57}$ & 44.084 & 0.082 & 1.938 & 56.15 & 184.95 & 333.43 & 7.96 \\
\hline $2015 \mathrm{GJ}_{57}$ & 43.477 & 0.027 & 1.328 & 82.03 & 40.14 & 85.44 & 7.69 \\
\hline $2015 \mathrm{GT}_{57}$ & 44.065 & 0.067 & 2.285 & 92.10 & 72.51 & 37.83 & 8.55 \\
\hline $2015 \mathrm{RP}_{280}$ & 45.495 & 0.096 & 3.489 & 232.41 & 45.34 & 83.23 & 7.51 \\
\hline $2015 \mathrm{VB}_{169}$ & 43.867 & 0.083 & 2.083 & 104.86 & 130.78 & 176.87 & 7.91 \\
\hline $2015 \mathrm{VB}_{171}$ & 43.677 & 0.083 & 2.391 & 83.55 & 172.08 & 155.30 & 7.92 \\
\hline $2015 \mathrm{VV}_{170}$ & 43.065 & 0.031 & 0.908 & 30.91 & 288.17 & 93.42 & 9.06 \\
\hline $2015 \mathrm{VW}_{168}$ & 42.539 & 0.045 & 1.954 & 96.71 & 199.17 & 112.82 & 8.29 \\
\hline
\end{tabular}

\subsection{Analysis of approaches of TNO and their orbits}

When assessing the age of pairs of small bodies in close orbits, it is interesting to compare the moments of the approach of orbits, lines of nodes and apsides, and the objects themselves in orbits. At the moment of pair formation, all estimated distances between orbits and bodies should be small. Let us compare the moments of the approach of orbits (see Tab. 3), nodes and pericenter of orbits, as well as objects in orbits (see Tab. 4) for six TNOs experiencing close 
Table 3. Minimum values of the metric $\varrho_{2}$ and the moments of their occurrence $t_{\varrho}$.

\begin{tabular}{lllr}
\hline \hline Binary TNO & TNO & $\varrho_{2 m i n}\left[\mathrm{AU}^{1 / 2}\right]$ & $t_{\varrho}$ [years] \\
\hline $2005 \mathrm{GD}_{187}$ & $2001 \mathrm{OG}_{109}$ & 0.036 & -2840 \\
$(275809) 2001 \mathrm{QY}_{297}$ & $2015 \mathrm{VB}_{169}$ & 0.062 & -259660 \\
$(469610) 2004 \mathrm{HF}_{79}$ & $1997 \mathrm{CT}_{29}$ & 0.056 & -53400 \\
$2005 \mathrm{GD}_{187}$ & $2015 \mathrm{VV}_{170}$ & 0.059 & -6417930 \\
$2002 \mathrm{VD}_{131}$ & $(505446) 2013 \mathrm{SP}_{99}$ & 0.056 & -9570 \\
$2004 \mathrm{HK}_{79}$ & $2015 \mathrm{VB}_{171}$ & 0.081 & -124000 \\
$2006 \mathrm{JV}_{58}$ & $2004 \mathrm{KF}_{19}$ & 0.057 & -246070 \\
$(275809) 2001 \mathrm{QY}_{297}$ & $2006 \mathrm{HB}_{123}$ & 0.063 & -22060 \\
$2003 \mathrm{QY}_{90}$ & $2002 \mathrm{CU}_{154}$ & 0.069 & -80050 \\
$2015 \mathrm{RP}_{280}$ & $2012 \mathrm{HE}_{85}$ & 0.067 & -40850 \\
$2000 \mathrm{CF}_{105}$ & $2015 \mathrm{GJ}_{57}$ & 0.102 & -13740 \\
$2003 \mathrm{UN}_{284}$ & $1996 \mathrm{TK}_{66}$ & 0.086 & -5006740 \\
$2015 \mathrm{VW}_{168}$ & $2013 \mathrm{UL}_{17}$ & 0.081 & -801640 \\
$2004 \mathrm{HK}_{79}$ & $2004 \mathrm{VB}_{131}$ & 0.079 & -8040 \\
$(505447) 2013 \mathrm{SQ}_{99}$ & $2004 \mathrm{HE}_{79}$ & 0.070 & -7297160 \\
$2004 \mathrm{HK}_{79}$ & $2015 \mathrm{GA}_{57}$ & 0.057 & -9421100 \\
$2005 \mathrm{GD}_{187}$ & $(420356) \mathrm{Praamzius}_{2}$ & 0.084 & -1157230 \\
$2004 \mathrm{PW}_{117}$ & $2015 \mathrm{GT}_{57}$ & 0.020 & -7254560 \\
$2002 \mathrm{VD}_{131}$ & $2002 \mathrm{CZ}_{224}$ & 0.107 & -1970310 \\
$2004 \mathrm{PW}_{117}$ & $2013 \mathrm{TL}_{172}$ & 0.056 & -7689270 \\
$(469610) 2004 \mathrm{HF}_{79}$ & $2013 \mathrm{UL}_{17}$ & 0.0057 & -4159900 \\
\hline \hline
\end{tabular}

Table 4. The closest approach moments $t_{r}$ of TNO in pairs under the condition $r_{\text {rel }}<10 R_{H}$.

\begin{tabular}{lllllll}
\hline \hline Binary TNO & TNO & $\begin{array}{l}r_{r e l} \\
{\left[R_{H}\right]}\end{array}$ & $\begin{array}{l}R_{H} \\
{\left[10^{3} \mathrm{~km}\right]}\end{array}$ & $\begin{array}{l}v_{\text {rel }} \\
{\left[V_{\text {esc }}\right]}\end{array}$ & $\begin{array}{l}V_{\text {esc }} \\
{\left[\mathrm{m} \mathrm{s}^{-1}\right]}\end{array}$ & $\begin{array}{l}t_{r} \\
{[\text { years }]}\end{array}$ \\
\hline (469610) 2004 $\mathrm{HF}_{79}$ & $1997 \mathrm{CT}_{29}$ & 8.6 & 439 & 213 & 0.26 & -3714950 \\
$2005 \mathrm{GD}_{187}$ & $2015 \mathrm{VV}_{170}$ & 4.5 & 307 & 232 & 0.25 & -3463500 \\
$2002 \mathrm{VD}_{131}$ & $(505446) 2013 \mathrm{SP}_{99}$ & 9.4 & 428 & 596 & 0.22 & -2230280 \\
$2006 \mathrm{JV}_{58}$ & $2004 \mathrm{KF}_{19}$ & 8.7 & 389 & 921 & 0.23 & -4696470 \\
$2000 \mathrm{CF}_{105}$ & $2015 \mathrm{GJ}_{57}$ & 6.4 & 356 & 684 & 0.23 & -4590180 \\
$2005 \mathrm{GD}_{187}$ & $(420356)$ Praamzius & 2.8 & 631 & 167 & 0.65 & -6882190 \\
\hline \hline
\end{tabular}

encounters. At the moment of pair formation, the conditions $t_{r} \approx t_{\varrho}, \Delta \Omega \approx 0$, and $\Delta g \approx 0$ must be met. The Tab. 5 gives the moments $t_{\Delta}$ corresponding to the condition $\Delta \Omega \approx 0$ and $\Delta g \approx 0$ for six pairs from the Tab. 4.

Pair TNOs (469610) 2004 $\mathrm{HF}_{79}-1997 \mathrm{CT}_{29}$. The minimum distance between TNOs is fixed at the moment of $t_{r}=-3714950$ years. The value of the metric $\varrho_{2}$ reaches its minimum at $t_{\varrho}=-53400$ years. The evolution of the metric $\varrho_{2}$ with time shows that the metric, on average, monotonically increases in 
Table 5. The moments $t_{\Delta}$ corresponding to the condition $\Delta \Omega \approx 0$ and $\Delta g \approx 0$.

\begin{tabular}{lll}
\hline \hline Binary TNO & TNO & $t_{\Delta}[\mathrm{Myr}]$ \\
\hline (469610) 2004 $\mathrm{HF}_{79}$ & $1997 \mathrm{CT}_{29}$ & -3.2 \\
$2005 \mathrm{GD}_{187}$ & $2015 \mathrm{VV}_{170}$ & -3.0 \\
& & -4.2 \\
& & -6.8 \\
& & $-9.4--9.0$ \\
$2002 \mathrm{VD}_{131}$ & $(505446) 2013 \mathrm{SP}_{99}$ & $-0.7--0.5$ \\
$2006 \mathrm{JV}_{58}$ & $2004 \mathrm{KF}_{19}$ & -0.7 \\
$2000 \mathrm{CF}_{105}$ & $2015 \mathrm{GJ}_{57}$ & -1.5 \\
$2005 \mathrm{GD}_{187}$ & $(420356)$ Praamzius & -5.6 \\
& & -6.4 \\
\hline \hline
\end{tabular}

the past from the moment of minimum. Analysis of the change in the differences in the longitudes of the ascending nodes $\Delta \Omega$ and the arguments of the pericenter $\Delta g$ shows that both differences are close to zero in the vicinity of the time 3.2 Myr in the past and in the present. Since the methods used give significantly different estimates of the pair's age, we did not find low relative-velocity close encounters of TNOs in the considered time interval, and we conclude that the age of the pair exceeds $10 \mathrm{Myr}$.

Pair TNOs $2005 G D_{187}-2015 V V_{170}$. The minimum distance between TNOs is fixed at the moment of $t_{r}=-3463500$ years. The value of the metric $\varrho_{2}$ reaches a minimum at $t_{\varrho}=-6417930$ years. The evolution of the metric $\varrho_{2}$ with time shows that the metric remains small for the entire considered interval. Its value does not exceed $0.13 \mathrm{AU}^{1 / 2}$. Analysis of the differences in the longitudes of the ascending nodes $\Delta \Omega$ and the arguments of the pericenter $\Delta g$ shows that both differences are close to zero in the vicinity of 3, 4.2, 6.8, 9.0-9.4 Myr in the past. The minimum distances between TNOs and between their orbits are achieved in the vicinity of the moments of the approach of the lines of nodes and apsides. However, we did not find low relative-velocity close encounters of TNOs in the considered time interval. Most likely, the age of the pair exceeds 10 Myr.

Pair TNOs 2002 $V D_{131}$ - (505446) $2013 S P_{99}$. The minimum distance between TNOs is fixed at the moment of $t_{r}=-2230280$ years. The value of the metric $\varrho_{2}$ reaches its minimum at $t_{\varrho}=-9570$ years. The evolution of the metric $\varrho_{2}$ with time shows that the distance between the orbits increases into the past, reaching $0.9 \mathrm{AU}^{1 / 2}$. Analysis of the change in the differences in the longitudes of the ascending nodes $\Delta \Omega$ and the arguments of the pericenter $\Delta g$ shows that both differences are close to zero about $0.5-0.7 \mathrm{Myr}$ ago. The methods used give significantly different estimates of the pair's age. We did not detect any low relative-velocity close encounters of TNOs. Most likely, the age of the pair exceeds 10 Myr. 
Pair TNOs $2006 J V_{58}-2004 K F_{19}$. The minimum distance between TNOs is fixed at the moment of $t_{r}=-4696470$ years. The value of the metric $\varrho_{2}$ reaches a minimum at $t_{\varrho}=-246070$ years. Starting from $1 \mathrm{Myr}$ ago, the metric $\varrho_{2}$ grows, reaching the value $0.7 \mathrm{AU}^{1 / 2}$ at the end of the considered interval. Analysis of the change in the differences in the longitudes of the ascending nodes $\Delta \Omega$ and the arguments of the pericenter $\Delta g$ shows that both differences are close to zero about $0.7 \mathrm{Myr}$ ago. The methods used give significantly different estimates of the pair's age. We did not detect any low relative-velocity close encounters of TNOs. Most likely, the age of the pair exceeds $10 \mathrm{Myr}$.

Pair TNOs $2000 \mathrm{CF}_{105}-2015 \mathrm{GJ}_{57}$. The minimum distance between TNOs is fixed at the moment of $t_{r}=-4590180$ years. The value of the metric $\varrho_{2}$ reaches a minimum at $t_{\varrho}=-13740$ years. Starting from 2 Myr ago, the metric $\varrho_{2}$ grows, reaching the value $0.5 \mathrm{AU}^{1 / 2}$ at the end of the considered interval. Analysis of the change in the differences in the longitudes of the ascending nodes $\Delta \Omega$ and the arguments of the pericenter $\Delta g$ shows that both differences are close to zero about 1.5 Myr ago. The methods used give significantly different estimates of the pair's age. We did not detect any low relative-velocity close encounters of TNOs. Most likely, the age of the pair exceeds $10 \mathrm{Myr}$.

Pair TNOs $2005 G D_{187}$ - (420356) Praamzius. The minimum distance between TNOs is fixed at the moment of $t_{r}=-6882190$ years. The value of the metric $\varrho_{2}$ reaches a minimum at $t_{\varrho}=-1157230$ years. Starting from 2 Myr ago, the metric $\varrho_{2}$ grows, reaching the value $0.25 \mathrm{AU}^{1 / 2}$ at the end of the considered interval. Analysis of the change in the differences in the longitudes of the ascending nodes $\Delta \Omega$ and the arguments of the pericenter $\Delta g$ shows that both differences are close to zero in the vicinity of 5.6 and $6.4 \mathrm{Myr}$ ago. The minimum distances between TNOs and between their orbits are achieved in the vicinity of the moments of the approach of the lines of nodes and apsides. However, we did not find low relative-velocity close encounters of TNOs in the considered time interval. Most likely, the age of the pair exceeds $10 \mathrm{Myr}$.

\section{Discussion and conclusions}

We performed a search for TNO pairs, in which one of the objects is binary, among all numbered objects and objects observed in more than one opposition. The measure of the proximity of the orbits was the Kholshevnikov metric $\varrho_{2}$. We selected 21 pairs of TNOs in close orbits to study the dynamic evolution and estimate the age of the pairs. All pairs belong to the dynamically cold population of the classical Kuiper belt. In this region, favorable conditions are formed for the preservation of close binary TNO systems (Nesvorný \& Vokrouhlický, 2019). However, at the same time, wide TNO binary systems disintegrate due to encounters with other objects (Campbell, 2021). The most probable source of TNO pairs, in which one of the objects is binary, is the decay of ternary and multiple TNO systems. 
The analysis of dynamic evolution based on nominal orbits over $10 \mathrm{Myr}$, carried out using three approaches: the search for the approaches of orbits, lines of nodes and apsides, the TNOs themselves in orbits, showed ambiguous results. As a rule, estimates of the age of pairs obtained by different methods gave significantly different estimates. This may indicate that the age of the pairs exceeds 10 Myr.

An approach based on the study of the probabilistic evolution of a pair of TNOs should play an important role in increasing the reliability of estimates of the age of TNO pairs. We plan to use this technic in our future studies.

The interval of $10 \mathrm{Myr}$ is relatively short for TNO because, during this time, objects of the classical Kuiper belt make only $33-36$ thousand periods in orbit. For comparison, young pairs in the main asteroid belt are pairs with an age of up to 2 Myr. During this time, asteroids make $400-600$ thousand the orbital periods. In the future, it is planned to increase the integration interval to 200 Myr. On such a long interval, the manifestation of stochastic properties of the TNO dynamic evolution is inevitable; therefore, the main methods used to estimate the age of pairs should be methods that estimate the distance between orbits, their nodes, and pericenters.

Acknowledgements. This work has been supported by the Russian Ministry of Science and Higher Education via the State Assignment Project FEUZ-2020-0038.

\section{References}

Benz, W. \& Asphaug, E., Catastrophic Disruptions Revisited. 1999, Icarus, 142, 5, DOI: $10.1006 /$ icar.1999.6204

Boehnhardt, H. 2004, Split comets, ed. M. C. Festou, H. U. Keller, \& H. A. Weaver, 301

Bottke, William F., J., Vokrouhlický, D., Rubincam, D. P., \& Nesvorný, D., The Yarkovsky and Yorp Effects: Implications for Asteroid Dynamics. 2006, Annual Review of Earth and Planetary Sciences, 34, 157, DOI: 10.1146/annurev.earth.34.031405.125154

Bowell, E., Hapke, B., Domingue, D., et al., Application of photometric models to asteroids. 1989, in Asteroids II, ed. R. P. Binzel, T. Gehrels, \& M. S. Matthews, $524-556$

Brown, M. E., Barkume, K. M., Ragozzine, D., \& Schaller, E. L., A collisional family of icy objects in the Kuiper belt. 2007, Nature, 446, 294, DOI: 10.1038/nature05619

Campbell, H., Stability and Formation of Ultra-Wide Kuiper Belt Binaries. 2021, in AAS/Division of Dynamical Astronomy Meeting, Vol. 53, AAS/Division of Dynamical Astronomy Meeting, 501.04

Chiang, E. I., A Collisional Family in the Classical Kuiper Belt. 2002, Astrophys. J. Lett., 573, L65, DOI: 10.1086/342089 
Chiang, E. I., Lovering, J. R., Millis, R. L., et al., Resonant and Secular Families of the Kuiper Belt. 2003, Earth Moon and Planets, 92, 49, DOI: 10.1023/B:MOON.0000031924.20073.d0

de la Fuente Marcos, C. \& de la Fuente Marcos, R., Far from random: dynamical groupings among the NEO population. 2016, Mon. Not. R. Astron. Soc., 456, 2946, DOI: $10.1093 / \mathrm{mnras} / \operatorname{stv} 2885$

de la Fuente Marcos, C. \& de la Fuente Marcos, R., Dynamically correlated minor bodies in the outer Solar system. 2018, Mon. Not. R. Astron. Soc., 474, 838, DOI: $10.1093 / \mathrm{mnras} / \mathrm{stx} 2765$

Deienno, R., Gomes, R. S., Walsh, K. J., Morbidelli, A., \& Nesvorný, D., Is the Grand Tack model compatible with the orbital distribution of main belt asteroids? 2016, Icarus, 272, 114, DOI: 10.1016/j.icarus.2016.02.043

Fernández, J. 2020, Introduction: The Trans-Neptunian zone: past, present and future, ed. D. Prialnik, M. A. Barucci, \& L. Young, 1-22

Gladman, B., Marsden, B. G., \& Vanlaerhoven, C. 2008, Nomenclature in the Outer Solar System, ed. M. A. Barucci, H. Boehnhardt, D. P. Cruikshank, A. Morbidelli, \& R. Dotson, 43

Granvik, M., Morbidelli, A., Vokrouhlický, D., et al., Escape of asteroids from the main belt. 2017, Astronomy and Astrophysics, 598, A52, DOI: 10.1051/0004$6361 / 201629252$

Grundy, W. M., Noll, K. S., Buie, M. W., et al., Mutual orbits and masses of six transneptunian binaries. 2009, Icarus, 200, 627, DOI: 10.1016/j.icarus.2008.12.008

Grundy, W. M., Noll, K. S., Nimmo, F., et al., Five new and three improved mutual orbits of transneptunian binaries. 2011, Icarus, 213, 678, DOI: 10.1016/j.icarus.2011.03.012

Grundy, W. M., Noll, K. S., Roe, H. G., et al., Mutual orbit orientations of transneptunian binaries. 2019, Icarus, 334, 62, DOI: 10.1016/j.icarus.2019.03.035

Grundy, W. M., Noll, K. S., Virtanen, J., et al., (42355) Typhon Echidna: Scheduling observations for binary orbit determination. 2008, Icarus, 197, 260, DOI: $10.1016 /$ j.icarus.2008.04.004

Jacobson, S. A., Multiple origins of asteroid pairs. 2016, in IAU Symposium, Vol. 318, Asteroids: New Observations, New Models, ed. S. R. Chesley, A. Morbidelli, R. Jedicke, \& D. Farnocchia, 55-65

Jacobson, S. A. \& Scheeres, D. J., Dynamics of rotationally fissioned asteroids: Source of observed small asteroid systems. 2011, Icarus, 214, 161, DOI: 10.1016/j.icarus.2011.04.009

Kavelaars, J., Jones, L., Gladman, B., Parker, J. W., \& Petit, J. M. 2008, The Orbital and Spatial Distribution of the Kuiper Belt, ed. M. A. Barucci, H. Boehnhardt, D. P. Cruikshank, A. Morbidelli, \& R. Dotson, 59

Kholshevnikov, K. V., Kokhirova, G. I., Babadzhanov, P. B., \& Khamroev, U. H., Metrics in the space of orbits and their application to searching for celestial objects 
of common origin. 2016, Mon. Not. R. Astron. Soc., 462, 2275, DOI: 10.1093/mnras/stw1712

Kholshevnikov, K. V., Shchepalova, A. S., \& Jazmati, M. S., On a quotient space of keplerian orbits. 2020, Vestnik St. Petersburg University: Mathematics, 53, 108, DOI: $10.1134 / \mathrm{S} 1063454120010045$

Kuznetsov, E. \& Safronova, V., Application of metrics in the space of orbits to search for asteroids on close orbits. 2018, Planet. Space Sci., 157, 22, DOI: $10.1016 /$ j.pss.2018.04.011

Kuznetsov, E. D., Al-Shiblawi, O. M., Gusev, V. D., \& Ustinov, D. S., Pairs of TransNeptunian Objects with Close Orbits. 2021, in Lunar and Planetary Science Conference, Lunar and Planetary Science Conference, 1859

Kuznetsov, E. D., Rosaev, A. E., Plavalova, E., Safronova, V. S., \& Vasileva, M. A., A Search for Young Asteroid Pairs with Close Orbits. 2020, Solar System Research, 54, 236, DOI: 10.1134/S0038094620030077

Kuznetsov, E. D. \& Vasileva, M. A., On New Members of Asteroid Clusters Similar to Asteroid Pairs. 2019, Meteoritics \& 8 Planetary Science, 54, A229, DOI: https://doi.org/10.1111/maps.13346

Lacerda, P. \& Jewitt, D. C., Densities of Solar System Objects from Their Rotational Light Curves. 2007, Astronomical Journal, 133, 1393, DOI: 10.1086/511772

Marcus, R. A., Ragozzine, D., Murray-Clay, R. A., \& Holman, M. J., Identifying Collisional Families in the Kuiper Belt. 2011, Astrophys. J., 733, 40, DOI: 10.1088/0004$637 \mathrm{X} / 733 / 1 / 40$

Nesvorný, D. \& Vokrouhlický, D., Binary survival in the outer solar system. 2019, Icarus, 331, 49, DOI: 10.1016/j.icarus.2019.04.030

Orbfit Consortium. 2011, OrbFit: Software to Determine Orbits of Asteroids, Astrophysics Source Code Library

Pravec, P., Fatka, P., Vokrouhlický, D., et al., Asteroid clusters similar to asteroid pairs. 2018, Icarus, 304, 110, DOI: 10.1016/j.icarus.2017.08.008

Pravec, P., Fatka, P., Vokrouhlický, D., et al., Asteroid pairs: A complex picture. 2019, Icarus, 333, 429, DOI: 10.1016/j.icarus.2019.05.014

Pravec, P. \& Vokrouhlický, D., Significance analysis of asteroid pairs. 2009, Icarus, 204, 580, DOI: 10.1016/j.icarus.2009.07.004

Pravec, P., Vokrouhlický, D., Polishook, D., et al., Formation of asteroid pairs by rotational fission. 2010, Nature, 466, 1085, DOI: 10.1038/nature09315

Rabinowitz, D., Schwamb, M. E., Hadjiyska, E., Rojo, P., \& Tourtellottee, S., A Tale of Two TNOs. in , EPSC-DPS Joint Meeting 2011, Vol. 2011, 1642

Rosaev, A. \& Plávalová, E., New members of Datura family. 2017, Planetary Space Science, 140, 21, DOI: 10.1016/j.pss.2017.01.017

Schunová, E., Jedicke, R., Walsh, K. J., et al., Properties and evolution of NEO families created by tidal disruption at Earth. 2014, Icarus, 238, 156, DOI: 10.1016/j.icarus.2014.05.006 
Sekanina, Z. \& Chodas, P. W., Origin of the Marsden and Kracht Groups of Sunskirting Comets. I. Association with Comet 96P/Machholz and Its Interplanetary Complex. 2005, Astrophys. J. Suppl. S., 161, 551, DOI: 10.1086/497374

Sekanina, Z. \& Chodas, P. W., Fragmentation Hierarchy of Bright Sungrazing Comets and the Birth and Orbital Evolution of the Kreutz System. II. The Case for Cascading Fragmentation. 2007, Astrophys. J., 663, 657, DOI: 10.1086/517490

Vokrouhlický, D. \& Nesvorný, D., Pairs of Asteroids Probably of a Common Origin. 2008, Astron. J., 136, 280, DOI: 10.1088/0004-6256/136/1/280

Vokrouhlický, D., Pravec, P., Durech, J., et al., Detailed Analysis of the Asteroid Pair (6070) Rheinland and (54827) 2001 NQ8. 2017, Astron. J., 153, 270, DOI: $10.3847 / 1538-3881 /$ aa72ea 